\title{
ISOMETRIC ISOMORPHISMS BETWEEN BANACH ALGEBRAS RELATED TO LOCALLY COMPACT GROUPS
}

\author{
F. GHAHRAMANI, A. T. LAU AND V. LOSERT
}

\begin{abstract}
Let $G_{1}, G_{2}$ be locally compact groups. We prove in this paper that if $T$ is an isometric isomorphism from the Banach algebra $\operatorname{LUC}\left(G_{1}\right)^{*}$ (the continuous dual of the Banach space of left uniformly continuous functions on $G_{1}$, equipped with Arens multiplication) onto $\operatorname{LUC}\left(G_{2}\right)^{*}$, then $T$ maps $M\left(G_{1}\right)$ onto $M\left(G_{2}\right)$ and $L^{1}\left(G_{1}\right)$ onto $L^{1}\left(G_{2}\right)$. We also prove that any isometric isomorphism from $L^{1}\left(G_{1}\right)^{* *}$ (second conjugate algebra of $L^{1}\left(G_{1}\right)$ ) onto $L^{1}\left(G_{2}\right)^{* *}$ maps $L^{1}\left(G_{1}\right)$ onto $L^{1}\left(G_{2}\right)$.
\end{abstract}

\section{INTRODUCTION AND PRELIMINARIES}

Let $G_{1}, G_{2}$ be locally compact groups. Let $M\left(G_{i}\right), i=1,2$, be the Banach algebra of regular Borel measures on $G_{i}$. A well-known result of B. E. Johnson [10] asserts that if $T$ is an isometric isomorphism from $M\left(G_{1}\right)$ onto $M\left(G_{2}\right)$, then $T$ maps $L^{1}\left(G_{1}\right)$ onto $L^{1}\left(G_{2}\right)$ (and hence $G_{1}$ and $G_{2}$ must be isomorphic by Wendel's theorem [21]).

In this paper we prove (Theorem 3.1(c)), among other things, that if $T$ is an isometric isomorphism from $L^{1}\left(G_{1}\right)^{* *}$ onto $L^{1}\left(G_{2}\right)^{* *}$, then $T$ maps $L^{1}\left(G_{1}\right)$ onto $L^{1}\left(G_{2}\right)$. This answers affirmatively a question raised in [4]. Theorem 3.1(c) was proved for abelian locally compact groups by Lau and Losert in [13], and for compact and discrete groups by Ghahramani and Lau in [4].

Let $G$ be a locally compact group. Let $C(G)$ denote the space of bounded continuous complex-valued functions on $G$ with the sup norm topology, and $\operatorname{LUC}(G)$ denote the closed subspace of bounded left uniformly continuous functions on $G$, i.e. all $f \in C(G)$ such that the map $x \mapsto l_{x} f$ from $G$ into $C(G)$ is continuous, where $\left(l_{x} f\right)(y)=f(x y), x, y \in G$. Then $\operatorname{LUC}(G)^{*}$ is a Banach algebra with the Arens multiplication defined by $\langle n m, f\rangle=\left\langle n, m_{l} f\right\rangle$, $n, m \in \operatorname{LUC}(G)^{*}, f \in \operatorname{LUC}(G)$, where $m_{l} f(x)=\left\langle m, l_{x} f\right\rangle, x \in G$. Furthermore, $M(G)$ may be identified with a closed subspace of $\operatorname{LUC}(G)^{*}$ by the natural embedding $\langle\mu, f\rangle=\int f(x) d \mu(x), f \in \operatorname{LUC}(G), \mu \in M(G)$. It was

Received by the editors November 1, 1988.

1980 Mathematics Subject Classification (1985 Revision). Primary 43A20.

Key words and phrases. Locally compact groups, uniformly continuous functions, grcup algebra, Arens multiplication, measure algebra, isometric isomorphisms, right identities.

The first two authors are supported by NSERC grants. 
shown by Grosser and Losert [7] that when $G$ is abelian, $M(G)$ is precisely the centre of $\operatorname{LUC}(G)^{*}$ (see also Lau [12]).

The organization of this paper is as follows. We prove in $\S 1$ (Theorem 1.6) that if $T$ is an isometric isomorphism from $\operatorname{LUC}\left(G_{1}\right)^{*}$ onto $\operatorname{LUC}\left(G_{2}\right)^{*}$, then $T$ maps $M\left(G_{1}\right)$ onto $M\left(G_{2}\right)$ and $L^{1}\left(G_{1}\right)$ onto $L^{1}\left(G_{2}\right)$. In $\S 2$ we study the set $\Lambda(G)$ of right identities with norm one in $L^{1}(G)^{* *}$ and the isometric embeddings $\Gamma_{E}$ of $M(G)$ into $L^{1}(G)^{* *}$ defined by S. McKilligan [16]. Finally we prove in $\S 3$ (and using results established in $\S \S 1$ and 2) that, if $T$ is an isometric isomorphism from $L^{1}\left(G_{1}\right)^{* *}$ onto $L^{1}\left(G_{2}\right)^{* *}$, then $T$ maps $L^{1}\left(G_{1}\right)$ to $L^{1}\left(G_{2}\right)$.

Throughout the paper, $G$ denotes a locally compact group with a fixed left Haar measure $\lambda$. Integration with respect to $\lambda$ will be denoted by $\int \cdots d x$. The spaces $L^{1}(G) \quad\left(=L^{1}(G, \lambda)\right)$ and $L^{\infty}(G) \quad\left(=L^{\infty}(G, \lambda)\right)$ are as defined in [8]. If $f$ and $g$ are measurable functions on $G$, then

$$
(f * g)(x)=\int_{G} f(y) g\left(y^{-1} x\right) d y,
$$

whenever this makes sense. If $f$ is any function defined on $G$, then for $x \in G$, the right (resp. left) translate of $f$ by $x$ will be denoted by $r_{x} f$ (resp. $l_{x} f$ ). We denote by $C_{00}(G)$ the functions in $C(G)$ with compact support and by $C_{0}(G)$ the functions in $C(G)$ which vanish at infinity.

We recall the definition for the (first) Arens product [1] (see also [3]) in the second conjugate of $L^{1}(G)$ : for $f \in L^{\infty}(G)$ and $\varphi \in L^{1}(G)$ let $f \varphi \in L^{\infty}(G)$ be defined by

$$
\langle f \varphi, \psi\rangle=\langle f, \varphi * \psi\rangle \quad\left(\psi \in L^{1}(G)\right) .
$$

For $m \in L^{1}(G)^{* *}$, let $m f \in L^{\infty}(G)$ be defined by $\langle m f, \varphi\rangle=\langle m, f \varphi\rangle$. Finally for $m, n \in L^{1}(G)^{* *}$, let $n m \in L^{1}(G)^{* *}$ be defined by $\langle n m, f\rangle=\langle n, m f\rangle$. It is easy to see that for $f \in L^{\infty}(G)$ and $\varphi \in L^{1}(G), f \varphi=\tilde{\varphi} * f$, where $\tilde{\varphi}(x)=\Delta\left(x^{-1}\right) \varphi\left(x^{-1}\right)$ and $\Delta$ denotes the modular function of the group [22]. Also if $f \in \operatorname{LUC}(G), m \in L^{1}(G)^{* *}$, then $m f \in \operatorname{LUC}(G)$ and $(m f)(x)=$ $m_{l}(f)(x)=\left\langle m, l_{x} f\right\rangle, x \in G$. (See [11, Lemma 3].)

A closed linear subspace $X$ of $C(G)$ is left introverted (see Day [2, p. 540]) if $l_{a}(X) \subseteq X$ for each $a \in G$, and for each $m \in X^{*}, f \in X$, the function $m_{l}(f)$ on $G$ defined by $m_{l}(f)(x)=m\left(l_{x} f\right), x \in G$, is also in $X$. In this case the Arens multiplication on $X^{*}$ defined by $\langle n m, f\rangle=\left\langle n, m_{l}(f)\right\rangle$ for each $f \in X, n, m \in X^{*}$ makes sense. Furthermore, $X^{*}$ with this multiplication is a Banach algebra (see $[2, \S 6])$. Examples of left introverted subspaces of $C(G)$ include $C_{0}(G)$, LUC $(G)$, and the space of almost periodic (resp. weakly almost periodic) functions on $G$. In the case of $C_{0}(G)^{*}=M(G)$, the multiplication on $M(G)$ is precisely the convolution of measures as defined in [8, p. 266]. Furthermore, $\operatorname{LUC}(G)$ is the maximal left introverted subspace of $C(G)[17$ and 18$]$. 


\section{ISOMETRIC ISOMORPHISMS ON $\operatorname{LUC}(G)^{*}$}

Let $C_{0}(G)^{\perp}=\left\{m \in \operatorname{LUC}(G)^{*} ; m(f)=0\right.$ for all $\left.f \in C_{0}(G)\right\}$.

1.1. Lemma. $\operatorname{LUC}(G)^{*}=C_{0}(G)^{\perp} \oplus M(G)$. If $m \in \operatorname{LUC}(G)^{*}$, and $m=m_{1}+\mu$ where $m_{1} \in C_{0}(G)^{\perp}, \mu \in M(G)$, then $\|m\|=\left\|m_{1}\right\|+\|\mu\|$. Furthermore, $C_{0}(G)^{\perp}$ is a closed ideal in $\operatorname{LUC}(G)^{*}$.

Proof. Clearly $C_{0}(G)^{\perp} \cap M(G)=\{0\}$. If $m \in \operatorname{LUC}(G)^{*}$, let $\mu$ denote the restriction of $m$ to $C_{0}(G)$. Let $\mu$ also denote the corresponding extension of $\mu$ to $\operatorname{LUC}(G)$. Then $m_{1}=m-\mu \in C_{0}(G)^{\perp}$ and $m=m_{1}+\mu$. To see that $\|m\|=\left\|m_{1}\right\|+\|\mu\|$, let $\varepsilon>0$; choose $h \in C_{00}(G)$ such that $\|h\| \leq 1$ and $\mu(h) \geq\|\mu\|-\varepsilon$. Let $F$ be a compact set such that $h(x)=0$ for all $x \notin F$. Let $V$ be an open set with compact closure such that $V \supseteq F$. Let $0 \leq g \leq 1$ such that $g \equiv 1$ on $F$ and $g(x)=0$ for all $x \notin V$. Let $k \in \operatorname{LUC}(G)$ such that $\|k\| \leq 1$ and $m_{1}(k) \geq\left\|m_{1}\right\|-\varepsilon$. Define $k^{\prime}=k-g k+h$. Then $m_{1}\left(k^{\prime}\right)=m_{1}(k)$ and $\left\|k^{\prime}\right\| \leq 1$. Furthermore, $\|\alpha(k-g k)+h\| \leq 1$ for any $\alpha \in \mathbf{C}$ with $|\alpha|=1$. By a proper choice of $\alpha$, one gets

$$
\begin{aligned}
\|\mu\| & \geq|\mu(\alpha(k-g k)+h)|=|\mu(k-g k)|+|\mu(h)| \\
& \geq|\mu(k-g k)|+\|\mu\|-\varepsilon .
\end{aligned}
$$

Hence $|\mu(k-g k)| \leq \varepsilon$ and

$$
\left|m\left(k^{\prime}\right)\right| \geq m_{1}\left(k^{\prime}\right)+\mu(h)-|\mu(k-g k)| \geq\left\|m_{1}\right\|+\|\mu\|-3 \varepsilon .
$$

So $\|m\| \geq\left\|m_{1}\right\|+\|\mu\|$.

To see that $C_{0}(G)^{\perp}$ is an ideal, let $h \in C_{0}(G), \varphi \in L^{1}(G)$. Then $h \varphi=$ $\tilde{\varphi} * h \in C_{0}(G)$. Hence if $n \in C_{0}(G)^{\perp}$, it follows that $\langle n h, \varphi\rangle=\langle n, h \varphi\rangle=0$, i.e. $n h=0$. Consequently $m n \in C_{0}(G)^{\perp}$ for all $m \in \operatorname{LUC}(G)^{*}$, i.e. $C_{0}(G)^{\perp}$ is a left ideal in $\operatorname{LUC}(G)^{*}$.

If $\mu \in M(G)$, then it is easy to see that $\mu h=h * \mu^{*}$ (where

$$
\left.\int f(t) d \mu^{*}(t)=\int \Delta\left(t^{-1}\right) f\left(t^{-1}\right) d \mu(t) \quad\left(f \in C_{0}(G)\right)\right) .
$$

In particular $\mu h \in C_{0}(G)$ for $h \in C_{0}(G)$. Hence $n \in C_{0}(G)^{\perp}$ implies $n \mu \in$ $C_{0}(G)^{\perp}$ (since $\left.\langle n \mu, h\rangle=\langle n, \mu h\rangle\right)$. Now if $m \in \operatorname{LUC}(G)^{*}$ is arbitrary, it can be written as $m=\mu+m_{1}$ with $\mu \in M(G), m_{1} \in C_{0}(G)^{\perp}$. If as above $n \in C_{0}(G)^{\perp}$, then $n m_{1} \in C_{0}(G)^{\perp}$, so $n m=n \mu+n m_{1} \in C_{0}(G)^{\perp}$. Thus, $C_{0}(G)^{\perp}$ is a right ideal. That completes the proof of the lemma.

1.2. Corollary. Let $m \in \operatorname{LUC}(G)^{*}$. Then, the following are equivalent.

(a) $m$ is invertible and $\|m\|=\left\|m^{-1}\right\|=1$.

(b) There exists $x \in G, \alpha \in \mathbf{C}$ with $|\alpha|=1$ such that $m=\alpha \delta_{x}$.

Proof. That (b) $\Rightarrow$ (a) is clear. To prove (a) $\Rightarrow$ (b) write $m=\mu+m_{1}$, $m^{-1}=\nu+m_{2}$ with $\mu, \nu \in M(G), m_{1}, m_{2} \in C_{0}(G)^{\perp}$. Then $\delta_{e}=\mu * \nu+$ $\left(\mu m_{2}+m_{1} \nu+m_{1} m_{2}\right)$ and the part in brackets belongs to $C_{0}(G)^{\perp}$, by Lemma 
1.1. Hence $\|\mu * \nu\|=\|\mu\|=\|\nu\|=1, m_{1}=m_{2}=0$ (again by Lemma 1.1). If $h \in C_{0}(G)$ satisfies $0 \leq h \leq 1$ and $h(e)=1$, then $1=\left\langle\delta_{e}, h\right\rangle=\langle\mu, \nu h\rangle$. Since $0 \leq|\nu h| \leq 1$, we conclude that $|\nu h(t)|=1$ for all $t \in \operatorname{supp} \mu$. Since $\nu h(t)=\int h(t s) d \nu(s)$, it follows that $h(t s)=1$ for all $t \in \operatorname{supp} \mu, s \in \operatorname{supp} \nu$. From this it follows that $(\operatorname{supp} \mu)(\operatorname{supp} \nu)=\{e\}$. In particular supp $\mu$ consists of a single point, i.e. $\mu=\alpha \delta_{x}$ for some $x \in G, \alpha \in \mathbf{C},|\alpha|=1$.

1.3. Remark. (a) Note that Corollary 1.2 may also be obtained as a consequence of Lemma 2 in [14].

(b) Let $X$ be a left introverted subspace of $C(G)$ containing $C_{0}(G)$. Then $M(G)$ may also be regarded as a closed subspace of $X^{*}$ by the isometric embedding: $\rho: M(G) \rightarrow X^{*}$, where $\rho(\mu)(f)=\int f(x) d \mu(x), f \in X, \mu \in M(G)$. In this case both Lemma 1.1 and Corollary 1.2 remain valid with $\operatorname{LUC}(G)$ replaced by $X$.

Let $\left\{m_{\alpha}\right\}$ be a net in $\operatorname{LUC}(G)^{*}$. We say that $m_{\alpha}$ converges to some $m \in$ $\operatorname{LUC}(G)^{*}$ strictly if $\left\|m_{\alpha} \phi-m \phi\right\| \rightarrow 0$, for all $\phi \in L^{1}(G)$.

1.4. Lemma. Let $G_{1}$ and $G_{2}$ be locally compact and let $T$ be an isometric isomorphism from $\operatorname{LUC}\left(G_{1}\right)^{*}$ onto $\operatorname{LUC}\left(G_{2}\right)^{*}$. Let $\left\{m_{\alpha}\right\}$ be a net in $M(G)$ converging strictly to $m \in M(G)$ and $\left\|m_{\alpha}\right\|=\|m\|=1$, then $T\left(m_{\alpha}\right)$ converges to $T(m)$ in the weak ${ }^{*}$-topology of $\operatorname{LUC}\left(G_{2}\right)^{*}$.

Proof. Let $n$ be a weak ${ }^{*}$-cluster point of $\left\{T\left(m_{\alpha}\right)\right\}$. By passing to a subnet, if necessary, we may assume that $T\left(m_{\alpha}\right) \rightarrow n$ in the $w^{*}$-topology. Let $\varphi \in L^{1}\left(G_{1}\right)$ be fixed. Since $\left\|m_{\alpha} \varphi-m \varphi\right\| \rightarrow 0$, it follows that $\left\|T\left(m_{\alpha}\right) T(\varphi)-T(m) T(\varphi)\right\| \rightarrow$ 0 . Hence for each $k \in \operatorname{LUC}\left(G_{2}\right)$,

$$
\langle T(m) T(\varphi), k\rangle=\lim _{\alpha}\left\langle T\left(m_{\alpha}\right) T(\varphi), k\right\rangle=\langle n, T(\varphi) k\rangle=\langle n T(\varphi), k\rangle,
$$

i.e. $T(m) T(\varphi)=n T(\varphi)$ or $m \varphi=T^{-1}(n) \varphi$, for all $\varphi \in L^{1}\left(G_{1}\right)$. Consequently, if $\varphi \in L^{1}\left(G_{1}\right), f \in \operatorname{LUC}\left(G_{1}\right)$,

$$
\langle m \varphi, f\rangle=\left\langle T^{-1}(n) \varphi, f\right\rangle .
$$

Hence $\langle m, \varphi f\rangle=\left\langle T^{-1}(n), \varphi f\right\rangle$. Consequently, $n$ agrees with $T^{-1}(n)$ on $C_{0}(G)$. Since $1=\|m\| \leq\left\|T^{-1}(n)\right\|=\|n\| \leq 1$, it follows that $m=T^{-1}(n)$ or $n=T(m)$ by Lemma 1 in [14].

Let $\tau: G_{1} \rightarrow G_{2}$ be a (topological) isomorphism of $G_{1}$ onto $G_{2}$ and let $\alpha: G_{1} \rightarrow \mathbf{T}$ (where $\mathbf{T}=\{\lambda \in \mathbf{C}:|\lambda|=1\}$ ) be a continuous character on $G_{1}$. Define $\tau_{\alpha}: C_{0}\left(G_{2}\right) \rightarrow C_{0}\left(G_{1}\right)$ by $\tau_{\alpha}(f)(x)=\alpha(x) f(\tau(x))$ for all $x \in G_{1}, f \in$ $C_{0}\left(G_{2}\right)$. Then $\tau_{\alpha}$ is an isometric isomorphism mapping $C_{0}\left(G_{2}\right)$ onto $C_{0}\left(G_{1}\right)$. Furthermore, $T_{\tau, \alpha}=\tau_{a}^{*}$ is an isometric algebra isomorphism from $M\left(G_{1}\right)$ onto $M\left(G_{2}\right)$ such that $T_{\tau, \alpha}\left(\delta_{x}\right)=\alpha(x) \delta_{\tau(x)}, x \in G_{1}$.

For each $\mu \in M\left(G_{1}\right)$, let $\mu^{\tau} \in M\left(G_{2}\right)$ be defined by

$$
\left\langle\mu^{\tau}, f\right\rangle=\int_{G_{1}} f(\tau(x)) d \mu(x), \quad f \in C_{0}\left(G_{2}\right) .
$$


Also let

$$
\hat{\mu}(\alpha)=\int_{G_{1}} \alpha(x) d \mu(x) .
$$

1.5. Lemma. Let $T$ be an isometric isomorphism from $\operatorname{LUC}\left(G_{1}\right)^{*}$ onto LUC $\left(G_{2}\right)^{*}$ such that $T\left(\delta_{x}\right)=T_{\tau, \alpha}\left(\delta_{x}\right)$ for each $x \in G_{1}$. Then $T(\mu)=\hat{\mu}(\alpha) \mu^{\tau}$ for each $\mu \in M\left(G_{1}\right)$. In particular $T$ maps $M\left(G_{1}\right)$ onto $M\left(G_{2}\right)$ in $\operatorname{LUC}\left(G_{2}\right)^{*}$ and $L^{1}\left(G_{1}\right)$ onto $L^{1}\left(G_{2}\right)$.

Proof. The equation

$$
T(\mu)=\hat{\mu}(\alpha) \mu^{\tau}
$$

clearly holds for all $\mu=\delta_{x}, x \in G_{1}$, and hence all convex combinations of all such measures. Let $\mu \geq 0$ and $\|\mu\|=1$. There exists a net $\mu_{\beta}=\sum_{i=1}^{n_{\beta}} \lambda_{i}^{\beta} \delta_{x_{i}}$ of convex combination of $\delta_{x}$ 's, $x \in G_{1}$, such that $\mu_{\beta}$ converges to $\mu$ in the $w^{*}$ topology. Since $\left\|\mu_{\beta}\right\|=\|\mu\|=1$ for each $\beta, \mu_{\beta}$ must converge to $\mu$ strictly (see [5 or 15]). Hence by Lemma 1.4, $T\left(\mu_{\beta}\right)$ must converge to $T(\mu)$ in the weak ${ }^{*}$-topology. Now the net $\hat{\mu}_{\beta}(\alpha) \mu_{\beta}^{\tau} \rightarrow \hat{\mu}(\alpha) \mu^{\tau}$ in the weak ${ }^{*}$-topology also. Hence (1) holds for all $\mu \geq 0,\|\mu\|=1$. Consequently (1) must hold for all $\mu \in M(G)$.

The last statement follows from [10]. However it also follows directly from the well-known fact that $L^{1}(G)$ can be identified with all $\mu \in M(G)$ such that the map $a \mapsto \delta_{a} * \mu$ from $G$ into $(M(G),\|\cdot\|)$ is continuous.

We are now ready to prove the main theorem of this section.

1.6. Theorem. Let $G_{1}$ and $G_{2}$ be locally compact groups and $T$ be an isometric isomorphism from $\mathrm{LUC}\left(G_{1}\right)^{*}$ onto $\mathrm{LUC}\left(G_{2}\right)^{*}$, then $T$ maps $M\left(G_{1}\right)$ onto $M\left(G_{2}\right)$ and $L^{1}\left(G_{1}\right)$ onto $L^{1}\left(G_{2}\right)$.

Proof. Indeed for each $x \in G_{1}, T\left(\delta_{x}\right)$ is invertible and $\left\|T\left(\delta_{x}\right)\right\|=\left\|T\left(\delta_{x}\right)^{-1}\right\|$ $=1$. Hence by Corollary 1.2 there exist $\alpha(x) \in \mathbf{C},|\alpha(x)|=1$ and $\gamma(x) \in G_{2}$ such that $T\left(\delta_{x}\right)=\alpha(x) \delta_{\gamma(x)}$. Clearly $\alpha$ is a character and $\gamma$ is an algebraic isomorphism of $G_{1}$ onto $G_{2}$. Furthermore, if $x_{i} \rightarrow x, x_{i}, x \in G_{1}$, then $\delta_{x_{i}} \rightarrow \delta_{x}$ strictly. Hence by Lemma $1.4 T\left(\delta_{x_{i}}\right) \rightarrow T\left(\delta_{x}\right)$ in the weak *topology of $\operatorname{LUC}\left(G_{2}\right)^{*}$. Consequently $\alpha\left(x_{i}\right) \rightarrow \alpha(x)$ and $\gamma\left(x_{i}\right) \rightarrow \gamma(x)$, i.e. both $\alpha$ and $\gamma$ are continuous. Hence $T\left(\delta_{x}\right)=T_{\tau, \alpha}\left(\delta_{x}\right)$ for each $x \in G_{1}$. The theorem now follows from Lemma 1.5.

1.7. Remark. Lemmas $1.4,1.5$ and Theorem 1.6 are valid when $\operatorname{LUC}\left(G_{i}\right)$, $i=1,2$ are replaced by left introverted subspaces $X_{i}$ of $C\left(G_{i}\right)$ containing $C_{0}\left(G_{i}\right)$ (see [14, Theorem 1]). When $X_{1}=C_{0}\left(G_{1}\right)$ and $X_{2}=C_{0}\left(G_{2}\right)$, this provides an alternative proof to the main result in [10].

$$
\text { 2. The embeddings } \Gamma_{E}: M(G) \rightarrow L^{1}(G)^{* *}
$$

Let $\Lambda(G)$ denote the set of weak ${ }^{*}$-cluster points of the canonical images of the bounded approximate identities, bounded by 1 , of $L^{1}(G)$ in $L^{1}(G)^{* *}$. We 
first observe that the set $\Lambda(G)$ coincides with the sets $K$ and $K_{1}$ considered in [9, Theorem 3.2] for compact groups:

2.1. Proposition. Let $E \in L^{1}(G)^{* *}$. The following are equivalent:

(a) $E \in \Lambda(G)$.

(b) $\|E\|=1$ and $E(f)=f(e)$ for all $f \in C_{0}(G)$.

(c) $E \geq 0, E \psi=\psi E=\psi$ for all $\psi \in L^{1}(G)$.

(d) $\|E\|=1$ and $E$ is a right identity of $L^{1}(G)^{* *}$.

Proof. (a) $\Rightarrow$ (b). If $E \in \Lambda(G)$, then $\|E\| \leq 1$. Let $\mu \in M(G)$ be the restriction of $E$ to $C_{0}(G)$. Then $\mu$ is the identity of $M(G)$ (by weak *-weak * continuity of multiplication in $M(G))$. So $\mu=\delta_{e}$, where $\delta_{e}(f)=f(e)$, $f \in C_{0}(G)$. Hence (b) holds.

(b) $\Rightarrow$ (c). Let $m$ denote the restriction of $E$ to $\operatorname{LUC}(G)$. Then $m(f)=$ $f(e)$ for all $f \in \operatorname{LUC}(G)$ by Lemma 1.1 and its proof. Hence $\|E\|=E(1)=1$. So $E \geq 0$ [20, p. 9]. Now if $\psi \in L^{1}(G), f \in L^{\infty}(G)$, then

$$
\langle E \psi, f\rangle=\langle E, \psi f\rangle=\langle E, f * \check{\psi}\rangle=(f * \check{\psi})(e)=\int f(t) \psi(t) d t=\langle\psi, f\rangle,
$$

i.e. $E \psi=\psi\left(\right.$ where $\left.\check{\psi}(t)=\psi\left(t^{-1}\right), t \in G\right)$. Similarly $\psi E=\psi$.

(c) $\Rightarrow(\mathrm{a})$. We first observe that $\|E\|=1$ (since $E(1)=E(\psi \cdot 1)=E \psi(1)=$ $\psi(1)=1$, when $\left.\psi \in L^{1}(G), \psi \geq 0,\|\psi\|_{1}=1\right)$. Let $P_{1}(G)$ denote all $\psi \in L^{1}(G), \psi \geq 0,\|\psi\|_{1}=1$. Let $\left\{\theta_{\alpha}\right\}$ be a net in $P_{1}(G)$ converging to $E$ in the weak ${ }^{*}$-topology. Then $\left\{\theta_{\alpha}\right\}$ is a weak approximate identity for $L^{1}(G)$. Then an argument similar to that in the proof of [2, Theorem 1, p. 524] shows that we can find a net $\left\{e_{\lambda}\right\}$ consisting of convex combinations of elements in $\left\{\theta_{a}\right\}$ such that

(i) $\left\|e_{\lambda} \psi-\psi\right\| \rightarrow 0$, for each $\psi \in L^{1}(G)$.

(ii) $\left\{e_{\lambda}\right\}$ is far out in $\left\{\theta_{\alpha}\right\}$, i.e. for each $\alpha_{0}$, there exists $\lambda_{0}$ such that if $\lambda \geq \lambda_{0}$, and $e_{\lambda}=\sum_{i=1}^{n} a_{i} \theta_{\alpha_{i}}, a_{i}>0, \sum_{i=1}^{n} a_{i}=1$, then each $\alpha_{i} \geq \alpha_{0}$. Then $\left\{e_{\lambda}\right\}$ is a left approximate identity in $L^{1}(G)$ converging in the weak ${ }^{*}$ topology of $L^{1}(G)^{* *}$ to $E$. Furthermore, $\left\{e_{\lambda}\right\}$ is also a weak right approximate identity in $L^{1}(G)$. Indeed, if $\psi \in L^{1}(G)$ and $f \in L^{\infty}(G)$, choose $\alpha_{0}$ such that $\left|\left\langle f, \psi \theta_{\alpha}-\psi\right\rangle\right|<\varepsilon$ for all $\alpha \geq \alpha_{0}$. Let $\lambda_{0}$ be as chosen in (ii). Then for all $\lambda \geq \lambda_{0}$,

$$
\begin{aligned}
\left|\left\langle f, \psi e_{\lambda}-\psi\right\rangle\right| & =\left|\left\langle f, \psi\left(\sum_{i=1}^{n} a_{i} \theta_{\alpha_{i}}\right)-\psi\right\rangle\right| \\
& \leq \sum_{i=1}^{n} a_{i}\left|\left\langle f, \psi \theta_{\alpha_{i}}-\psi\right\rangle\right|<\varepsilon\|f\| .
\end{aligned}
$$

Again, repeating the argument in the proof of [2, Theorem 1, p. 524], we can find a net $\left\{f_{\mu}\right\}$ consisting of convex combinations of elements in $\left\{e_{\lambda}\right\}$ such that 
(i) $\left\{f_{\mu}\right\}$ is a right approximate identity of $L^{1}(G)$.

(ii) $)^{\prime}\left\{f_{\mu}\right\}$ is far out in $\left\{e_{\lambda}\right\}$.

Necessarily, $\left\{f_{\mu}\right\} \subseteq P_{1}(G)$ and is also a left approximate identity of $L^{1}(G)$ converging in the weak ${ }^{*}$-topology of $L^{1}(G)^{* *}$ to $E$ by (ii) ${ }^{\prime}$.

(b) $\Rightarrow$ (d). If $E \in \Lambda(G)$, then $E$ is a right identity of $L^{1}(G)^{* *}$ by (a).

$(\mathrm{d}) \Rightarrow(\mathrm{b})$. Let $\theta$ denote the restriction of $E$ to $C_{0}(G)$. Then $\theta$ is a right identity in $C_{0}(G)^{*}$. It suffices to show $\theta$ is also a left identity. Let $\left\{e_{j}\right\}$ denote a bounded weak right approximate identity in $L^{1}(G)$ converging to $E$ in the weak *-topology. Let $f \in C_{0}(G)$. Then $f=g \psi$ for some $g \in C_{0}(G)$, $\psi \in L^{1}(G)$ (by Cohen's factorization theorem). Hence for each $m \in C_{0}(G)^{*}$,

$$
\begin{aligned}
\langle\theta m, f\rangle & =\lim _{j}\left\langle m, f e_{j}\right\rangle=\lim _{j}\left\langle m, g \psi e_{j}\right\rangle \\
& =\lim _{j}\left\langle m, g\left(\psi e_{j}\right)\right\rangle=\langle m, g \psi\rangle=\langle m, f\rangle,
\end{aligned}
$$

since $\varphi \mapsto\langle m, g \varphi\rangle$ defines a bounded linear functional on $L^{1}(G)$.

2.2. Remark. $\Lambda(G)$ does not change if one uses (weak) approximate identities, bounded by 1 , in the definition. It also does not change if one uses weak ${ }^{*}$-cluster points of positive bounded approximate identities in $L^{1}(G)$.

Let $E=w^{*}-\lim e_{j}$, where $\left(e_{j}\right)$ is a bounded approximate identity bounded by 1 . For $\mu \in M(G)$, let $\rho_{\mu}: L^{1}(G) \rightarrow L^{1}(G)$ be defined by $\rho_{\mu}(\nu)=\nu * \mu$, and let $\Gamma_{E}(\mu)=\rho_{\mu}^{* *}(E)$, where $\rho_{\mu}^{* *}$ is the second adjoint of $\rho_{\mu}$. Then

2.3. Proposition. (i) $\left\langle\Gamma_{E}(\mu), f\right\rangle=\int f d \mu(f \in \operatorname{LUC}(G), \mu \in M(G))$.

(ii) $\Gamma_{E}(\mu)=\mu$, if $\mu \in L^{1}(G)$.

(iii) $\left\langle\Gamma_{E}(\mu) f, \varphi\right\rangle=\langle\mu, \tilde{\varphi} * f\rangle \quad\left(f \in L^{\infty}(G), \varphi \in L^{1}(G), \mu \in M(G)\right)$. In particular, $\Gamma_{E}(\mu) f=\rho_{\mu}^{*} f$, for each $f \in L^{\infty}(G)$.

(iv) $\Gamma_{E}\left(\delta_{x}\right) f=r_{x} f\left(f \in L^{\infty}(G), x \in G\right)$, where $\delta_{x}$ is the Dirac measure at $x$.

(v) $\Gamma_{E}$ is an isometric embedding of the algebra $M(G)$ into $L^{1}(G)^{* *}$, which extends the canonical embedding of $L^{1}(G)$ into $L^{1}(G)^{* *}$.

Proof. (i) Let $\mu \in M(G)$ and $f \in \operatorname{LUC}(G)$. Then by a version of Cohen's factorization theorem $[8,32.45(\mathrm{~b})]$, there exists $g \in L^{1}(G)$ and $h \in L^{\infty}(G)$ such that $f=g * h$. Hence, with $\tilde{\varphi}(x)=\Delta\left(x^{-1}\right) \varphi\left(x^{-1}\right)$, we have

$$
\left\langle\Gamma_{E}(\mu), f\right\rangle=\lim _{j}\left\langle f, e_{j} * \mu\right\rangle=\lim _{j}\left\langle\tilde{e}_{j} * f, \mu\right\rangle=\langle f, \mu\rangle
$$

since $\left\{\tilde{e}_{j}\right\}$ is also a bounded approximate identity of $L^{1}(G)$ [22, Lemma 3.3], $\left\|\tilde{e}_{j} * f-f\right\|_{\infty} \rightarrow 0$, by another application of Cohen's factorization theorem.

(ii) follows directly from

$$
\left\langle\Gamma_{E}(\mu), f\right\rangle=\lim _{j}\left\langle f, e_{j} * \mu\right\rangle .
$$


(iii) We have

$$
\begin{aligned}
\left\langle\Gamma_{E}(\mu) f, \varphi\right\rangle & =\left\langle\Gamma_{E}(\mu), f \varphi\right\rangle=\left\langle\Gamma_{E}(\mu), \tilde{\varphi} * f\right\rangle=\langle\mu, \tilde{\varphi} * f\rangle=\langle\varphi \mu, f\rangle \\
& =\left\langle\rho_{\mu}(\varphi), f\right\rangle=\left\langle\varphi, \rho_{\mu}^{*}(f)\right\rangle
\end{aligned}
$$

by part (i).

(iv) follows from (iii) with $\mu=\delta_{x}$ and a direct computation.

(v) From the definition of $\Gamma_{E}(\mu)$ it follows that

$$
\left\|\Gamma_{E}(\mu)\right\|=\left\|\rho_{\mu}^{* *}(E)\right\| \leq\left\|\rho_{\mu}^{* *}\right\|\|E\|=\|\mu\| .
$$

This together with (i) shows that $\mu \mapsto \Gamma_{E}(\mu)$ is obviously linear. To prove that it is multiplicative we note that for $\mu, \nu \in M(G)$ and $f \in L^{\infty}(G)$,

$$
\begin{aligned}
\left\langle\Gamma_{E}(\mu) \Gamma_{E}(\nu), f\right\rangle & =\left\langle\Gamma_{E}(\mu), \Gamma_{E}(\nu) f\right\rangle=\left\langle\rho_{\mu}^{* *}(E), \rho_{\nu}^{*}(f)\right\rangle \\
& =\left\langle E, \rho_{\mu}^{*} \rho_{\nu}^{*}(f)\right\rangle=\left\langle E, \rho_{\mu * \nu}^{*} f\right\rangle \\
& =\left\langle\Gamma_{E}(\mu * \nu), f\right\rangle \quad \text { by (iii). }
\end{aligned}
$$

2.4. Proposition. (i) $E_{2} \Gamma_{E_{1}}(\mu)=\Gamma_{E_{2}}(\mu)$, for any $E_{1}, E_{2} \in \Lambda(G)$, and $\mu \in$ $M(G)$.

(ii) A measure $\mu$ belongs to $L^{1}(G)$ if and only if $\Gamma_{E_{1}}(\mu)=\Gamma_{E_{2}}(\mu)$, for any $E_{1}, E_{2} \in \Lambda(G)$.

Proof. (i) Suppose $h \in L^{\infty}(G)$. Then

$$
\left\langle E_{2} \Gamma_{E_{1}}(\mu), h\right\rangle=\left\langle E_{2}, \Gamma_{E_{1}}(\mu) h\right\rangle=\left\langle E_{2}, \rho_{\mu}^{*}(h)\right\rangle=\left\langle\rho_{\mu}^{* *}\left(E_{2}\right), h\right\rangle=\left\langle\Gamma_{E_{2}}(\mu), h\right\rangle,
$$

by (iii) of Proposition 2.4.

(ii) The "only if" part being obvious, we assume that $\mu \notin L^{1}(G)$. We then may (and do) assume that $\mu$ is real and $\mu \neq 0$. We will construct two bounded approximate identities $\left(e_{i}\right)$ and $\left(f_{j}\right)$ both bounded by 1 such that for a $w^{*}$ cluster point $E_{1}$ of $\left(e_{i}\right)$ and a $w^{*}$-cluster point $E_{2}$ of $\left(f_{j}\right), \Gamma_{E_{1}}(\mu) \neq \Gamma_{E_{2}}(\mu)$. By [19, Theorem 2], there exists a continuous function $f$ such that the function $h: x \mapsto \int f(x y) d \mu(y)$ is not (equal almost everywhere to) a function continuous at the identity $e$. We can also assume that $f$, and hence $h$, is real. We may further assume that for each neighbourhood $V$ of $e$ there are sets $A, B \subseteq V$ of positive Haar measure with $h \geq 1$ on $A$ and $h \leq 0$ on $B$. By the method of the proof of $\left[9\right.$, Lemma 2.3] there exists bounded approximate identities $\left(e_{i}\right)$ and $\left(f_{i}\right)$ of $L^{1}(G)$ bounded by 1 , with $\left\langle e_{i}, h\right\rangle \geq 1$ and $\left\langle f_{j}, h\right\rangle \leq 0$. Now let $E_{1}=w^{*}-\lim e_{i}^{\prime}$ and $E_{2}=w^{*}-\lim f_{j}^{\prime}$ where $\left(e_{i}^{\prime}\right)$ is a subnet of $\left(e_{i}\right)$ and $\left(f_{j}^{\prime}\right)$ is a subnet of $\left(f_{j}\right)$. Then

$$
\left\langle\Gamma_{E_{1}}(\mu), f\right\rangle=\lim _{i}\left\langle f, \mu * e_{i}^{\prime}\right\rangle=\lim _{i}\left\langle e_{i}^{\prime}, h\right\rangle \geq 1,
$$

while

$$
\left\langle\Gamma_{E_{2}}(\mu), f\right\rangle=\lim _{j}\left\langle f, \mu * f_{j}^{\prime}\right\rangle=\lim _{j}\left\langle f_{j}^{\prime}, h\right\rangle \leq 0 .
$$


2.5. Proposition. Let $m \in L^{1}(G)^{* *}$ and $E \in \Lambda(G)$. Then the following are equivalent:

(a) $m=\Gamma_{E}(\mu)$, for some $\mu \in M(G)$.

(b) As a functional $m$ is an extension of $\mu \in C_{0}(G)^{*}$ with $\|m\|=\|\mu\|$ and $E m=m$.

Proof. (a) $\Rightarrow$ (b) follows from parts (i) and (v) of Proposition 2.3 together with part (i) of Proposition 2.4. To prove (b) $\Rightarrow$ (a) let $m$ be an extension of $\mu$ with $\|m\|=\|\mu\|$. Then the norm of the restriction of $m$ to $\operatorname{LUC}(G)$ will also be equal to $\|m\|$. Then from [14, Lemma 1] it follows that for $f \in$ $\operatorname{LUC}(G),\langle m, f\rangle=\int f d \mu$. Hence by Proposition 2.3(i) $\langle m, f\rangle=\left\langle\Gamma_{E}(\mu), f\right\rangle$ for every $f \in \operatorname{LUC}(G)$. Now if $E$ is the $w^{*}$-limit of $\left(e_{j}\right)$, then from $E m=m$ we have

$$
\begin{aligned}
\langle m, f\rangle & =\langle E m, f\rangle=\langle E, m f\rangle=\lim _{j}\left\langle e_{j}, m f\right\rangle \\
& \left.=\lim _{j}\left\langle m f, e_{j}\right\rangle=\lim _{j}\left\langle m, f e_{j}\right\rangle=\lim _{j}\left\langle\mu, f e_{j}\right\rangle \quad \text { (since } f e_{j} \in \operatorname{LUC}(G)\right) \\
& =\lim _{j}\left\langle\mu, \tilde{e}_{j} * f\right\rangle=\lim _{j}\left\langle f, e_{j} * \mu\right\rangle=\left\langle\Gamma_{E}(\mu), f\right\rangle .
\end{aligned}
$$

In the following propositions the canonical image of $L^{1}(G)$ in $L^{1}(G)^{* *}$ will be denoted by the same symbol.

2.6. Proposition. Let $\Delta(G)=\bigcap E L^{1}(G)^{* *}$, where $E$ ranges in $\Lambda(G)$. Then $\Delta(G)$ is a closed right ideal of $L^{1}(G)^{* *}$ containing $L^{1}(G)$. Furthermore, $L^{1}(G)$ is an ideal in $\Delta(G)$ if and only if $G$ is compact, in which case $\Delta(G)=L^{1}(G)$.

Proof. Since for each $E \in \Lambda(G), E^{2}=E$ (by Proposition 2.1(d)), each $E L^{1}(G)^{* *}$ is a closed right ideal, whence $\Delta(G)$ is a closed right ideal. If $G$ is a compact group, then an argument similar to the one of $[9,3.3$, v] shows that $\Delta(G)=L^{1}(G)$. Suppose conversely that $L^{1}(G)$ is an ideal in $\Delta(G)$. Let $m \in L^{1}(G)^{* *}$ and $\psi \in L^{1}(G)$. Then $\psi m \in \Delta(G)$. Let $\left(\varphi_{\alpha}\right)$ be a bounded approximate identity of $L^{1}(G)$. Then $\varphi_{\alpha} \psi m \rightarrow \psi m$, in norm. So $\psi m \in L^{1}(G)$. Therefore, $L^{1}(G)$ is a right ideal in $L^{1}(G)^{* *}$. Hence $G$ is a compact group [6].

2.7. Proposition. The intersection of all $\Gamma_{E}(M(G)$ ) when $E$ ranges in $\Lambda(G)$ is equal to $L^{1}(G)$.

Proof. Let $\Omega$ denote the intersection of all $\Gamma_{E}(M(G))$, where $E$ ranges in $\Lambda(G)$. Suppose $m \in \Omega$, and let $E_{1}$ and $E_{2}$ belong to $\Lambda(G)$. Then for some $\mu, \nu \in M(G), m=\Gamma_{E_{1}}(\mu)=\Gamma_{E_{2}}(\nu)$. Hence $\Gamma_{E_{1}}(\mu)=E_{1} \Gamma_{E_{1}}(\mu)=E_{1} \Gamma_{E_{2}}(\nu)=$ $\Gamma_{E_{1}}(\nu)$, by Proposition $2.4(\mathrm{i})$. Hence $\mu=\nu$, and we have $\Gamma_{E_{1}}(\mu)=\Gamma_{E_{2}}(\mu)$ for every $E_{1}$ and $E_{2}$ in $\Lambda(G)$. From Proposition 2.4(ii), it now follows that $\mu \in L^{1}(G)$.

Let $E \in \Lambda(G)$ and let $\pi_{E}$ be the map which associates to any functional in $E L^{1}(G)^{* *}$ its restriction to $\operatorname{LUC}(G)$. Then $\pi_{E}$ is an isometric isomorphism from $E L^{1}(G)^{* *}$ onto $\operatorname{LUC}(G)^{*}$ (see [4]). 
2.8. Proposition. Let $E \in \Lambda(G)$. For each $\mu \in M(G)$, we have $\pi_{E}^{-1}(\mu)=$ $\Gamma_{E}(\mu)$.

Proof. Let $m \in L^{1}(G)^{* *}$ be an extension of $\mu$. Let $\left\{e_{j}\right\}$ be an approximate identity in $L^{1}(G)$ bounded by 1 converging to $E$ in the weak ${ }^{*}$-topology (see the proof of Proposition 2.1). Then for each $f \in L^{\infty}(G)$,

$$
\begin{aligned}
\left\langle\Gamma_{E}(\mu), f\right\rangle & =\lim _{j}\left\langle\mu, \tilde{e}_{j} * f\right\rangle=\lim _{j}\left\langle m, f e_{j}\right\rangle \\
& =\lim _{j}\left\langle m f, e_{j}\right\rangle=\langle E, m f\rangle=\langle E m, f\rangle,
\end{aligned}
$$

i.e. $\Gamma_{E}(\mu) \in E L^{1}(G)^{* *}$. Since $\Gamma_{E}(\mu)$ extends $\mu$ by Proposition 2.3(i), $\pi_{E}\left(\Gamma_{E}(\mu)\right)=\mu$, i.e. $\Gamma_{E}(\mu)=\pi_{E}^{-1}(\mu)$.

\section{ISOMETRIC ISOMORPHISMS ON $L^{1}(G)^{* *}$}

We are now ready to prove our next main result.

3.1. Theorem. Let $G_{1}$ and $G_{2}$ be locally compact groups and let $T$ be an isometric isomorphism from $L^{1}\left(G_{1}\right)^{* *}$ onto $L^{1}\left(G_{2}\right)^{* *}$. Then

(a) $T\left(\Lambda\left(G_{1}\right)\right)=\Lambda\left(G_{2}\right)$.

(b) For each $E \in \Lambda\left(G_{1}\right)$, there exists a continuous character $\alpha: G_{1} \rightarrow \mathbf{T}$ and a bicontinuous isomorphism $\tau: G_{1} \rightarrow G_{2}$ such that for each $\mu \in M\left(G_{1}\right)$

$$
T\left(\Gamma_{E}(\mu)\right)=\hat{\mu}(\alpha) \Gamma_{T(E)}\left(\mu^{\tau}\right) .
$$

(c) $T$ maps $L^{1}\left(G_{1}\right)$ onto $L^{1}\left(G_{2}\right)$.

Proof. (a) follows immediately from Proposition 2.1(d).

(b) Let $E \in \Lambda\left(G_{1}\right)$. Let $\tilde{T}=\pi_{T(E)} \circ T \circ \pi_{E}^{-1}$. Then $\tilde{T}$ is an isometric isomorphism from $\operatorname{LUC}\left(G_{1}\right)^{*}$ onto $\operatorname{LUC}\left(G_{2}\right)^{*}$ (see [4]). So by the proof of Lemma 1.5, there exist a continuous character $\alpha$ on $G_{1}$ and a bicontinuous isomorphism $\tau: G_{1} \rightarrow G_{2}$ such that $\tilde{T}(\mu)=\hat{\mu}(\alpha) \mu^{\tau}$, for each $\mu \in M(G)$. In particular,

$$
T \circ \pi_{E}^{-1}(\mu)=\hat{\mu}(\alpha) \pi_{T(E)}^{-1}\left(\mu^{\tau}\right) .
$$

So $T\left(\Gamma_{E}(\mu)\right)=\hat{\mu}(\alpha) \Gamma_{T(E)}\left(\mu^{\tau}\right)$ by Proposition 2.8.

(c) follows from (b) and Proposition 2.3(ii).

For each $m \in L^{1}(G)^{* *}$, let $Q_{m}$ denote the map from $L^{1}(G)^{* *} \rightarrow L^{1}(G)^{* *}$ defined by $Q_{m}(n)=m n, n \in L^{1}(G)^{* *}$.

3.2. Corollary. Let $G_{1}$ and $G_{2}$ be locally compact groups and let $T$ be an isometric isomorphism from $L^{1}(G)^{* *}$ onto $L^{1}\left(G_{2}\right)^{* *}$. Let $m \in L^{1}\left(G_{1}\right)^{* *}$. Then $Q_{m}$ is weak ${ }^{*}$-weak ${ }^{*}$ continuous if and only if $Q_{T(m)}$ is weak ${ }^{*}$-weak ${ }^{*}$ continuous.

Proof. This follows from Theorem 1 in [13] and Theorem 3.1 above.

3.3. Remark. Note that if $G_{1}$ and $G_{2}$ are abelian, then $Q_{m}$ is weak ${ }^{*}$-weak ${ }^{*}$ continuous if and only if $m$ is in the centre of $L_{1}(G)^{* *}$ (see [13, Lemma 7]). 
Hence in this case Corollary 3.2 holds even when $T$ is an algebraic isomorphism.

\section{REFERENCES}

1. R. Arens, The adjoint of a bilinear operation, Proc. Amer. Math. Soc. 2 (1951), 939-948.

2. M. M. Day, Amenable semigroups, Illinois J. Math. 1 (1957), 509-544.

3. J. Duncan and S. A. R. Hosseiniun, The second dual of a Banach algebra, Proc. Roy. Soc. Edinburgh Sect. A 84 (1979), 309-325.

4. F. Ghahramani and A. T. Lau, Isometric isomorphisms between the second conjugate algebras of the group algebras, Bull. London Math. Soc. 20 (1988), 342-344.

5. E. Granirer and M. Leinert, On some topologies which coincide on the unit sphere of the Fourier algebra $B(G)$ and of the measure algebra $M(G)$, Rocky Mountain J. Math. 11 (1981), 459-472.

6. M. Grosser, $L^{1}(G)$ as an ideal in its second dual space, Proc. Amer. Math. Soc. 73 (1979), 3633-3634.

7. M. Grosser and V. Losert, The norm-strict bidual of a Banach algebra and the dual of $C_{u}(G)$, Manuscripta Math. 45 (1984), 127-146.

8. E. Hewitt and K. A. Ross, Abstract harmonic analysis, vols. I \& II, Springer-Verlag, Berlin, 1963 and 1970.

9. N. Isik, J. S. Pym and A. Ülger, The second dual of the group algebra of a compact group, J. London Math. Soc. 35 (1987), 135-148.

10. B. E. Johnson, Isometric isomorphisms of measure algebras, Proc. Amer. Math. Soc. 15 (1964), 186-188.

11. A. T. Lau, Operators which commute with convolutions on subspaces of $L_{\infty}(G)$, Colloq. Math. 39 (1978), 351-359.

12. __ Continuity of Arens multiplication on the dual space of bounded uniformly continuous functions on locally compact groups and topological semigroups, Math. Proc. Cambridge Philos. Soc. 99 (1986), 273-283.

13. A. T. Lau and V. Losert, On the second conjugate algebra of $L^{1}(G)$ of a locally compact group, J. London Math. Soc. 37 (1988), 464-470.

14. A. T. Lau and K. McKennon, Isomorphisms of locally compact groups and Banach algebras, Proc. Amer. Math. Soc. 79 (1980), 55-58.

15. K. McKennon, Multipliers, positive functionals, positive-definite functions, and FourierStieltjes transforms, Mem. Amer. Math. Soc. No. 111 (1971).

16. S. A. McKilligan, On the representation of the multiplier algebras of some Banach algebras, J. London Math. Soc. 6 (1973), 399-402.

17. P. Milnes and J. S. Pym, Counterexample in the theory of continuous functions on topological groups, Pacific J. Math. 66 (1976), 205-209.

18. T. Mitchell, Topological semigroups and fixed points, Illinois J. Math. 14 (1970), 630-641.

19. L. Pigno, A multiplier theorem, Pacific J. Math. 34 (1970), 755-757.

20. S. Sakai, $C^{*}$-algebras and $W^{*}$-algebras, Springer-Verlag, Berlin and New York, 1971.

21. J. G. Wendel, Left centralizers and isomorphism of group algebras, Pacific J. Math. 2 (1952), 251-261.

22. J. C. S. Wong, Topologically stationary locally compact groups and amenability, Trans. Amer. Math. Soc. 144 (1969), 351-363.

Department of Mathematics, University of Manitoba, Winnipeg, Manitoba, Canada R3T 2 N2

Department of Mathematics, University of Alberta, Edmonton, Alberta, Canada T6G 2G1

Mathematisches Institut der Universität, Universitat Wien, A-1090 Wien, Austria 